\title{
SOME RESULTS FOR THE COMMUTATORS \\ OF GENERALIZED HAUSDORFF OPERATOR
}

\author{
AmJAD Hussain AND AMnA AJAIB
}

Abstract. In this paper, we study commutator of generalized Hausdorff operator on function spaces. We mainly discuss the continuity criteria for such commutator operator when the symbol functions are either from central- $B M O$ or Lipschitz class of functions.

Mathematics subject classification (2010): 42B35, 26D15, 42B30, 46E30.

Keywords and phrases: Generalized Hausdorff operators, commutators, Morrey space, Herz-Morrey space, homogeneous Triebel Lizorkin spaces.

\section{REFERENCES}

[1] A. BAernstein And E. SAWyer, Embedding and multiplier theorems for $H^{p}\left(\mathbb{R}^{n}\right)$, Mem. Amer. Math. Soc., 53, (1985), 1-82.

[2] V. Burenkov, H. Guliyev And V. Guliyev, Necessary and sufficient conditions for the bounndedness of fractional maximal operators in local Morrey type spaces, J. Comput. Appl. Math., 208, (2007), 280-301.

[3] V. BuREnKoV And E. LiflyAnd, On the boundedness of Hausdorff operators on Morrey-type spaces, Eurasian Math. J., 8, (2017), 97-104.

[4] J. Chen, D. FAn And J. Li, Hausdorff operators on function spaces, Chin. Ann. Math., 33, (2012), 537-556.

[5] J. Chen, S. HE AND X. ZHU, Boundedness of Hausdorff operators on the power weighted Hardy spaces, Appl. Math., 32, (2017), 462-476.

[6] J. ChEn, D. FAn AND S. WANG, Hausdorff operators on Eucleadian spaces, Appl. Math., 28, (2013), 548-564.

[7] J. CHEN AND X. ZHU, Boundedness of multidimensional Hausdorff operators on $H^{1}\left(\mathbb{R}^{n}\right)$, J. Math. Anal. Appl., 409, (2014), 428-434.

[8] M. Christ And L. Grafakos, Best constants for two nonconvolution inequalities, Proc. Amer. Math. Soc., 123, (1995), 1687-1693.

[9] G. GAO AND H. JiA, Boundedness of commutators of high dimensional Hausdorff operator, J. Function Spaces Appl., 2012, (2012):54120.

[10] G. Gao, X. Wu, A. Hussain And G. ZhaO, Some esimates for Hausdorff operators, J. Math. Inequal., 9, (2015), 641-651.

[11] G. GaO And Y. ZHOng, Some inequalities for Hausdorff operators, Math. Ineq. Appl., 17, (2014), 1061-1078.

[12] L. Grafakos, Classical Fourier Analysis, second edition, Graduate Texts in Mathematics, 249 , Springer, New York, 2008.

[13] Y. GuO, Boundedness of some operators on non-homogeneous spaces, Master Degree Dissertation, Beijing Normal University.

[14] J. GUO AND F. ZHAO, Some q-inequalities for Hausdorff operators, Front. Math. China, 12, (2017), 879-889.

[15] K. Ho, Hardy Littlewood Pólya inequalities and Hausdorff operator on Block spaces, Math. Inequal. Appl., 19, (2016), 697-707.

[16] H. Hung, L. Ky And T. QuAng, Norm of the Hausdorff Operator on the Real Hardy Space $H^{1}(\mathbb{R})$, Complex Anal. Oper. Theory, 12, (2018), 235-245. 
[17] A. Huss AIn AND A. AJAIB, Some weighted inequalities for Hausdorff operators and commutators, J. Inequal. Appl., 2018, (2018)6, 19 pages.

[18] A. HuSSAIN AND M. AhMED, Weak and strong type estimates for the commutators of Hausdorff operator, Math. Inequal. Appl., 20, (2017), 49-56.

[19] A. Hussain AND G. GAO, Some new estimates for the commutators of n-dimensional Hausdorff operator, Appl. Math., 29, (2014), 139-150.

[20] A. Hussain AND G. GAO, Multidimensional Hausdorff operators and commutators on Herz-type spaces, J. Ineq. Appl., 2013, (2013)594, 12 pages.

[21] Y. Kanjin, The Hausdorff operators on the real Hardy spaces $H^{p}(\mathbb{R})$, Studia Math., 148, (2001), $37-45$.

[22] A. LeRner AND E. LiflyAnd, Multidimensional Hausdorff operators on the real Hardy spaces, Jour. Austr. Math. Soc., 83, (2007), 79-86.

[23] E. LiflyAnd, Boundedness of multidimensional Hausdors operators on $H^{1}\left(\mathbb{R}^{n}\right)$, Acta Sci. Math. (Szeged), 74, (2008), 845-851.

[24] E. Liflyand, Hausdorff operators on Hardy spaces, Eurasian Math. J., 4, (2013), 101-141.

[25] E. LifLYAND AND A. MIYACHI, Boundedness of the Hausdorff operators in $H^{p}$ spaces, $0<p<1$, Stud. Math., 194, (2009), 279-292.

[26] E. LifLyAND AND F. MóRECZ, The Hausdorff operator is bounded on the real Hardy space $H^{1}(\mathbb{R})$, Proc. Amer. Math. Soc., 128, (2000), 1391-1396.

[27] S. Lu And D. YAng, Herz-type Sobolev and Bessel potential spaces and their applications, Sci. China Ser. A, 40, (1997), 113-129.

[28] S. Lu, D. YAng And G. Hu, Herz Type Spaces and Their Application, Science Press-Beijing, China, 2008.

[29] C. Morrey, On the solutions of quasi-linear elliptic partial differential equations, Trans. Amer. Math. Soc., 43, (1938), 126-166.

[30] M. Paluszynski, Characterization of Besov spaces via the commutator operator of Coifman, Rochberg and Weiss, Ind. Univ. Math. J., 44, (1995), 1-18.

[31] J. RUAN AND D. FAn, Hausdorff operators on the power weighted Hardy spaces, J. Math. Anal. Appl., 433, (2016), 31-48.

[32] J. RuAn AND D. FAn, Hausdorff operators on weighted Herz-type Hardy spaces, Math. Inequal. Appl., 19, (2016), 565-587.

[33] J. RuAn, D. FAN, AND Q. Wu, Weighted Herz space estimates for Hausdorff operators on the Heisenberg group, Banach J. Math. Anal., 11, (2017), 513-535.

[34] X. WU, Necessary and sufficient conditions for generalized Hausdorff operators and commutators, Ann. Funct. Anal., 6, (2015), 60-72. 